\title{
The cognitive and psychological sequelae of uncomplicated aneurysm surgery
}

\author{
R S Maurice-Williams, J R Willison, R Hatfield
}

\begin{abstract}
Many patients are left with psychological symptoms after surgery for a ruptured intracranial aneurysm. Often the reason for these symptoms is not clear. A prospective study was carried out of 27 patients who were in a good (Grade 1 or 2) condition before operation to identify the origin of such symptoms and discover whether the basic techniques of aneurysm surgery could lead to serious psychological sequelae even in the absence of any specific complication. Each patient was given a modified psychometric assessment just before surgery and at the time of discharge from hospital. One year later a full psychometric and social assessment was carried out. Even a temporary worsening of psychometric performance did not occur unless there had been some specific surgical or post-operative problem. Five patients showed worsening of psychometric performance in the immediate post-operative period but by one year, only two of the 27 patients showed any abnormalities on formal psychometric evaluation; in both, clear reasons were evident. Although the majority of patients reported minor psychological symptoms, these had not hindered full functional recovery, and we doubt whether they had any organic basis. It is concluded that aneurysm surgery does not, itself, threaten higher intellectual function unless some specific complication occurs.
\end{abstract}

After operations for ruptured intracranial aneurysms, many patients are left with changes of intellect, affect, or personality, even in the absence of any gross neurological deficit. Although it seems likely that these symptoms reflect a degree of cerebral damage, it is often not clear when such damage has occurred. Brain injury may result from events which take place before and after surgery as well as those which occur at the time of operation.

One possibility which does not seem to have been considered previously is that the basic techniques of aneurysm surgery may involve a risk of damage to the brain even in the absence of any specific complication, for even the most straight forward operation involves induced hypotension, sustained brain retraction and the dissection of basal structures. The situation could be analogous to that in cardiac surgery using an external bypass, where it has been discovered that many patients who come through surgery without any problems apparent at the time are left with neurological and psychological changes purely as the result of the techniques inseparable from surgery of this type..$^{1-3}$

Although there is considerable literature on the psychological changes seen after aneurysm operations, previous investigators have confined psychometric assessment to the period after operation, generally at long term follow up. In an attempt to separate the effects of surgery from the effects of cerebral injuries sustained before the operation we have, for the first time, subjected patients to psychometric assessment immediately before and after operation as well as at follow up. Our findings suggest that aneurysm surgery by itself does not lead to any detectable impairment of higher cerebral function in the absence of some specific surgical or postoperative complication.

\section{Method}

1) Patients studied

Over a period of 18 months, from September 1985 to February 1987, we carried out a prospective study of those patients in our unit who were to have surgery for a ruptured intracranial aneurysm and who were, before operation, clinically stable and in a good condition, that is, in grade 1 or 2 according to the Hunt and Hess criteria. ${ }^{4}$

During this period, 31 patients met these criteria. Twenty seven of these were studied. Of the other four patients, two were inadvertently omitted from the study, while two patients who had come from overseas for treatment were omitted deliberately. These patients could not be fully assessed psychologically because they did not speak English and it was uncertain whether they would be able to return for follow up.

\section{2) Clinical information}

As each patient was admitted, detailed clinical information was collected and the patient's clinical course before and after surgery was recorded in detail. At the time of surgery the following information was recorded: total operating time, brain retraction time, the duration and degree of deliberately induced hypotension, and the occurrence of any mishap or untoward event during the operation.

\section{3) Methods of treatment}

All the patients were operated on by one of two 
consultant neurosurgeons (RSM-W or KWL) Both surgeons used similar surgical techniques employing the operating microscope and fixed brain retraction in every case. Temporary occlusion of an artery proximal to the aneurysm was not used, but induced hypotension was. The only difference between the surgeons was that for one surgeon the total operating time and the brain retraction time tended to be shorter although this surgeon routinely employed a greater degree of hypotension during surgery than did the other.

Both before and after operation a deliberate attempt was made to keep up the blood volume by maintaining a high fluid intake. Antifibrinolytic and hypotensive agents were not given. Anticonvulsants were started at the time of operation, or earlier if the patient had already had a fit.

\section{4) Functional assessment}

Each patient was seen at between two to four months after surgery and a full assessment was carried out at approximately one year postoperatively (12-16 months). At this latter time, in addition to the final psychometric assessment and a neurological examination the following questions were asked:

a) Has the patient returned to his/her preillness level of work and/or function in daily life?

b) Does the patient feel that his/her mental state/capacity is as it was before the illness?

c) Does the nearest relative/friend feel that his/ her mental state has changed in any way?

d) Has the patient any symptoms of a psychological nature not present before the illness?

\section{5) Psychometric assessment}

Each patient was assessed a) on the day before operation, b) before discharge home, never less than seven days after surgery, c) approximately one year after surgery.

Before operation and before discharge each patient was given a battery of eight basic psychological tests designed so that they could be administered to a patient confined to bed. To eliminate any practice effect, two versions of the test battery were drawn up, each patient being given a different version before and after operation. Each test battery consisted of the following:

1) Naming objects from line drawings (20 items)

2) Comprehension of descriptions of objects ( 8 items)

3) Wechsler Digit Span: forwards and backwards

4) Binet Cube Counting Test (10 items)

5) Fragmented Letters Perception Test (20 items)

6) Recognition Memory for Faces (25 items)

7) Recognition Memory for Words (25 items)

8) Word Fluency (2 categories: time taken to generate 10 names from each).

At the one year follow up a rather fuller assessment was carried out consisting of:

1) The original test battery in the version the patient had been given pre-operatively.

2) A set of speed tests which combine to give a quotient similar in construction and meaning to the intelligence quotient.

3) A memory test which involved both object recall and spatial location sensitive to right/left hemisphere differences.

4) A set of "intellectual" tasks to assess frontal lobe functioning.

The psychometric assessments were carried out by Dr J Willison. The functional and neurological assessments at one year were performed by one of the two consultant neurosurgeons. Although the latter may have been aware of the clinical events which had occurred, they did not know the results of the psychometric tests, and in any case, the functional assessment consisted of the results of standard questions put to the patients.

\section{Results}

\section{1) Patient characteristics}

The 27 patients comprised 12 men and 15 women ranging in age from 20-65. Their occupations could be classified as: Professional 3, Managerial/Technical/Secretarial 7, Skilled Manual Workers 4, Unskilled/Housewives 13.

Only one patient had previously received treatment for a psychological illness (depression). Ten patients had had a preceding physical illness which might have had a bearing on their capacity for recovery after intracranial surgery. Seven patients had evidence of generalised vascular disease as indicated by hypertension and angina or intermittent claudication, two had had previous surgery for intracranial aneurysms (one clipping, one common carotid artery ligation) and one patient had had two episodes of cerebral concussion.

The ruptured aneurysms were located at the following sites: anterior communicating artery 14, middle cerebral artery 6 , internal carotid artery 6 , posterior inferior cerebellar artery 1 , Three patients had additional non ruptured aneurysms.

\section{2) Clinical course}

a) COURSE BEFORE SURGERY

At the time of admission 20 patients were judged to be in Grades 1 or 2, seven were in Grade 3. By the time of operation all were in Grades 1 and 2. No patient re-bled before operation but five patients experienced episodes of neurological deterioration from which they recovered without apparent ill effect. Two of these episodes were thought to be caused by raised CSF pressure, three to delayed cerebral ischaemia ("spasm").

\section{b) HYDROCEPHALUS}

Seven patients had evidence at some stage of obstruction of the CSF pathways as indicated by CT scanning or raised pressure on lumbar puncture. In five patients this became apparent before operation and resolved with lumbar drainage of cerebrospinal fluid. Two patients required the insertion of ventricular shunts after surgery. In one of these patients hydrocephalus was diagnosed as a result of intellectual deterioration eight months after the aneurysm had been clipped. This patient has required several subsequent shunt revisions. 


\section{c) EPILEPSY}

Three patients each had a single epileptic fitbefore operation, two days after surgery, and six weeks later.

\section{3) Surgical characteristics}

Twenty-six aneurysms were clipped, one was wrapped with muslin. The time from the last haemorrhage to operation ranged from five to 18 days (between days nine to 14 for 22 patients). In the 26 operations for supratentorial aneurysms, the right cerebral hemisphere was retracted in 16 patients, the left in 10 .

\section{4) Events during surgery}

a) TOTAL OPERATING TIME

This ranged from 70 to 300 minutes.

b) BRAIN RETRACTION TIME

This ranged from 11 to 240 minutes. In 90 patients it exceeded 60 minutes and in four of these it was greater than 120 minutes.

\section{C) PERIOD OF DELIBERATELY INDUCED HYPOTENSION}

This ranged from five to 180 minutes. It exceeded 60 minutes in seven patients and in two of these it exceeded 120 minutes.

\section{d) LOWEST SYSTOLIC BLOOD PRESSURE}

This ranged from 15 to $110 \mathrm{~mm} \mathrm{Hg}$. It fell below $80 \mathrm{~mm} \mathrm{Hg}$ in 15 patients and below $60 \mathrm{~mm} \mathrm{Hg}$ in four.

\section{e) UNTOWARD EVENTS DURING SURGERY}

There were no cases of brain swelling or laceration during surgery. In five patients the aneurysm bled while it was being dissected out. In two patients the aneurysm clip occluded a normal artery as well as the aneurysm neck, a state of affairs confirmed by post-operative angiography. In one of these, occlusion of the left ophthalmic artery led to no neurological deficit. In the other, occlusion of the right anterior choroidal artery caused a transient left hemiparesis; CT scanning showed a small area of capsular ischaemia. In another patient it was thought that perioperative trauma to perforating vessels arising from the middle cerebral artery was responsible for a severe contralateral hemiparesis.

\section{5) Immediate post-operative course}

No patient died and 15 patients recovered from surgery without any evident disturbance of intellectual or neurological function at any time.

On recovering from anaesthesia, seven patients were either drowsy or confused or had a focal neurological deficit. In six cases these disturbances resolved within a few days, often

Table 1 Outcome at 12-16 months after surgery $(n=27)$

\begin{tabular}{lcc}
\hline & Yes & No \\
\hline a) Return to pre-morbid level of function & 24 & 3 \\
b) Neurologically intact (other than cranial palsy) & 24 & 3 \\
c) Psychologically unchanged (relative's assessment) & 20 & 7 \\
d) Psychologically unchanged (patient's assessment) & 16 & 11 \\
e) Absence of psychological symptoms & 12 & 15 \\
\hline
\end{tabular}

within hours of operation but one patient who had a severe left hemiparesis after the wrapping of a right middle cerebral artery aneurysm has been left with a permanent slight clumsiness of the left hand.

A further six patients made a good initial recovery after operation but developed a delayed drowsiness or focal neurological deficit while in hospital. In one patient this was caused by a subdural hygroma which resolved spontaneously; in the remaining patients the aetiology was thought to be cerebrovascular spasm. One of these patients was left with a persistent right hemiparesis and dysphasia, in the remaining patients the neurological worsening was mild and transient.

\section{6) Neurological and functional status at follow} $u p$

This is shown in table 1. The different methods of assessment are presented so that the better outcome is presented in the left hand column. It will be seen that the proportion of patients in the "best outcome" group varied according to the method of assessment. Thus 24 patients ( 89 per cent) returned to their pre-morbid level of function or had no neurological deficit while at the other extreme only 12 (44 per cent) were left with no psychological symptoms at all.

The three patients with residual neurological signs had respectively a slight clumsiness of the left hand, a slight cerebellar-type ataxia of the left limbs, and a marked right hemiparesis with dysphasia. A further two patients had cranial nerve abnormalities-bilateral anosmia in one, unilateral impairment of central visual acuity and optic atrophy in the other.

Residual psychological symptoms mentioned by the patients were varied: irritability, impairments of memory and concentration, depression, dizziness, and headaches. Psychological changes noticed by the patients' next of kin included forgetfulness, irritability, depression and disinhibition. There were only 11 patients ( 40 per cent) who had no neurological deficit, were leading a normal life and were completely free of all psychological changes or symptoms whether noticed by themselves or their relatives.

The three patients who had not returned to their pre-illness functional level comprised a former nursing sister of 54 left with a dysphasia and right hemiparesis who is now attending a rehabilitation centre, a housewife of 60 who has not resumed full household duties because of the effects of hydrocephalus which has required repeated shunt revisions, and a medical research worker of 47 who returned to work for some months after surgery but then resigned and became unemployed after friction with his colleagues. It seemed that even before his illness there had been serious conflicts at work and these had been aggravated by a slight increase of his irascibility after surgery.

Five of the patients with residual psychological symptoms but no neurological deficit were at the time of follow up receiving medication (anti-convulsants or sedatives) which might have accounted for their symptoms of irritability, tiredness or depression. 
Table 2 Mean (SD) scores on basic test battery

\begin{tabular}{|c|c|c|c|c|}
\hline & Max score & Pre-operative & Post-operative & 1 year \\
\hline $\begin{array}{l}\text { Naming } \\
\text { Comprehension } \\
\text { Digit span } \\
\text { Cubes } \\
\text { Letters } \\
\text { Faces Memory } \\
\text { Words Memory } \\
\text { Fluency (seconds) }\end{array}$ & $\begin{array}{r}20 \\
8 \\
17 \\
10 \\
20 \\
25 \\
25 \\
-\end{array}$ & $\begin{array}{c}18 \cdot 9(1 \cdot 4) \\
7 \cdot 3(0 \cdot 9) \\
10 \cdot 5(2 \cdot 2) \\
8 \cdot 7(2 \cdot 1) \\
18 \cdot 8(1 \cdot 8) \\
22 \cdot 7(2 \cdot 6) \\
23 \cdot 0(3 \cdot 0) \\
69 \cdot 4(28 \cdot 6)\end{array}$ & $\begin{array}{c}18 \cdot 5(2 \cdot 0) \\
7 \cdot 2(1 \cdot 0) \\
10 \cdot 8(2 \cdot 2) \\
8 \cdot 5(2 \cdot 0) \\
18 \cdot 4(2 \cdot 6) \\
22 \cdot 5(2 \cdot 8) \\
22 \cdot 4(3 \cdot 9) \\
76 \cdot 1(36 \cdot 7)\end{array}$ & $\begin{array}{r}18.8(3.9) \\
7.6(0.7) \\
11.2(2.9) \\
9.0(1.7) \\
19.7(0.9) \\
23.7(1.9) \\
24.5(0.9) \\
47.0(21)\end{array}$ \\
\hline
\end{tabular}

\section{7) Results of psychometric assessment}

\section{a) THE BASIC TEST BATTERY}

Mean scores for each test both before and after operation and at the one year follow up are shown in table 2 . A reported measures analysis of variances was carried out on the results of each test. There were no significant differences between the mean scores pre-operatively and at discharge for any of the eight tests. At one year, the mean scores were improved for two tests compared with the pre-operative baseline, on Digit Span $(p<0.03)$ and on fluency $(p<0.01)$. The results for faces and words memory tests at one year nearly reached conventional significance levels (both $p<0.07$ ).

These of course are mean scores. Within the total of 27 patients there were a number who could be identified as performing worse after surgery. Eleven patients had at least one test where the post-operative performance was down by more than four points (tests 1 to 7) or 20 seconds (test 8 ). However, for seven of these 11 patients the worsening was for one test only and can probably be disregarded. Two patients performed considerably worse on only two tests each, leaving a further two who were worse after surgery on at least half the tests. In addition, a further patient could not be tested at all after operation because of aphasia.

Of this total of five patients who could be said to be clearly worse at the time of discharge on psychometric assessment, four had recovered to their pre-operative status on the test battery administered at the one year follow up leaving the one patient who was severly dysphasic. In the three of these five patients who were most affected a clear reason for the worsening could be found: delayed ischaemia of the left cerebral hemisphere, inadvertent occlusion of the right anterior choroidal artery, and very prolonged (240 minutes) fixed retraction of the dominant hemisphere). Of the two patients who performed worse on only two tests, one had postoperative raised CSF pressure which resolved on lumbar puncture; in the other patient no reason for the worsening was evident.

Three patients performed poorly on the preoperative assessment despite being assessed as being in Grades 1 or 2 from a clinical point of view, that is, they appeared to be grossly intellectually intact. These patients performed no worse at the post-operative assessment and all three had fully recovered by the time the test battery was repeated at one year.

\section{b) ONE YEAR ASSESSMENT}

There were only two patients in whom any abnormalities could be detected on either the basic test battery or the more extended psychometric assessment. The mean (SD) speed quotient was $99 \cdot 25(10 \cdot 8)$ compared with an expected score of 100 (15), the mean scale score for the WAIS. Similarities were $9.5(2.5)$ compared with an expected score of $10.0(3.0)$.

The two patients whose performances were impaired were the patient with aphasia and a right hemiparesis from delayed post-operative ischaemia and the patient who proved to have late-onset hydrocephalus requiring a shunt: The latter patient had shown no worsening after surgery on the basic test battery but had declined subsequently.

\section{Discussion}

It was Storey (1967) who first drew attention to the psychiatric sequelae of sub-arachnoid haemorrhage. He assessed 231 survivors of a ruptured aneurysm at between six months and six years after the event and found that 55 per cent were left with psychological symptoms. He thought that in most patients the symptoms originated from cerebral damage but that in some it resulted from anxiety or depression without any evidence of organic disturbance. ${ }^{5}$

Since then, numerous papers have been published on the subject of psychological changes after sub-arachnoid haemorrhage and/ or aneurysm surgery. ${ }^{6-17}$ The symptoms vary in severity, from psychotic or confusional states to mild changes in cognition, memory, affect and personality not dissimilar to those seen after a closed head injury. ${ }^{12}{ }^{13}$ These changes may be relatively subtle but nevertheless sufficient to prevent a resumption of normal life. ${ }^{7}$

Such symptoms often occur in patients with no detectable neurological abnormalities and who may otherwise be regarded as having done well after treatment. Differences in their reported incidence may reflect the assiduity with which they have been sought. ${ }^{914}$ The cause of the symptoms may not be clear. Brain damage may occur before operation either at the time of hydrocephalus or delayed cerebral ischaemia. Ljunggren suggested that the haemorrhage itself may cause a diffuse encephalopathy analogous to that resulting from traumatic concussion. ${ }^{7}$ In other patients damage may result from some mishap at the time of surgery or from some post-operative complication.

Previous studies have relied on psychometric evaluation after treatment. For the first time, we have tested patients before operation as well. We were concerned to investigate the possibility that aneurysm surgery might involve some risk of cerebral damage even in the absence of any specific complication. It is known that certain types of aneurysm operation may lead to cognitive changes as a result of focal cerebral damage. Thus with anterior communicating aneurysms, persistent amnesic symptoms are more common if the mode of treatment is occlusion of one anterior cerebral artery or isolation of the anterior communicating artery by clipping each end than if the neck of the aneurysm itself is clipped. ${ }^{121317}$ Nevertheless, it is not known whether the basic techniques of current aneurysm surgery carries some risk of causing cerebral damage. 
Our study involved some limitations. First, it was clearly impossible to perform meaningful psychometric assessment on patients who are confused, obtunded or dysphasic before operation. We therefore confined our study to patients in grades 1 and 2 , that is, those who had no focal neurological deficit and no impairment of conscious level. While most aneurysm operations are carried out on patients in these grades a proportion are performed on patients in a poorer condition and it is possible that straightforward surgery does involve a risk of causing cerebral damage in such higher grades even though this may not be the case for the stable patient without any neurological deficit.

Second, even in these selected cases, it was not practicable to carry out a full psychometric evaluation before operation. We used a battery of tests especially designed for bedbound patients. Some of these tests had a "ceiling", easily reached by many patients and hence not sufficient to detect all impairment of intellectual function. The fact that the results on two tests improved significantly at one year, and came close to significance on two others, however, demonstrates that a ceiling effect did not confound all the data. The tests were repeated before discharge but any "practice" effect was eliminated by using a different battery so that no patient repeated the same test twice. The fuller psychometric assessment at the one year follow up should have been sufficient to detect any significant residual changes.

A third limitation is the timing of surgery in this series. Of recent years there has been an increasing tendency for surgeons to operate on good condition patients in the first day or two after the haemorrhage. None of our patients was operated on at such an early stage, and it is possible that our findings would not apply to such patients who in theory might be more susceptible to the effects of basic aneurysm surgery technique than those patients whose condition had been stable for some while.

Our findings were reassuring for the aneurysm surgeon. In three of our 27 patients the pre-operative psychometry revealed abnormalities of intellectual functioning even though the patients were judged to be normal from a clinical point of view. Five of the 27 patients showed a clear worsening on psychometric testing immediately after surgery but in four of these the operative or post-operative course had been characterised by some problem or complication. Only one patient whose course had been straightforward showed worsening and then on only two of the eight tests. By one year, only two of the 27 patients showed any psychological impairment on full testing. One of these patients was the only one in the series who was left with any substantial neurological deficit-dysphasia and a right hemiparesis as the result of delayed post-operative ischaemia. The other patient had not shown any worsening at the pre-discharge psychometric assessment but had deteriorated by one year because of the development of communicating hydrocephalus.

We conclude that aneurysm surgery as presently practised does not by itself involve any risk of detectable psychological impairment unless there has been a specific problem or complication associated with the surgery. Nevertheless, there remains the fact that several patients and their relatives reported psychological symptoms and changes at the time of follow up in the absence of any detectable abnormalities on a fairly full psychometric evaluation. Twenty four of the 27 patients had returned to their pre-morbid level of function but there were 11 with relatively minor psychological changes. If one disregards the two patients who showed abnormalities on formal psychometric evaluation and for whom clear reasons for these abnormalities could be found one is left with nine of 25 patients ( $36 \%$ ) who felt psychologically altered and 13 of 25 patients $(52 \%)$ who had residual psychological symptoms. We could find no relationship between the occurrence of such changes or symptoms and the site or side of the aneurysm, the age of the patient, the previous medical history, and the degree of hypotension or the period of brain retraction at operation or per-operative rupture of the aneurysm. In five of the patients the presence of residual symptoms may have been related to the fact that they were taking anticonvulsant or psychotrophic drugs. In one of these patients the complaints were almost certainly fictitious - she complained of intractable depression since operation and stated that she had been completely well before surgery, although there was in fact a long preceding history of psychiatric treatment for depression!

There are three possible explanations for the minor residual symptoms in the remaining patients. The first is that these complaints reflect a minimal degree of cerebral damage, too slight to be detected even by the fuller psychological testing carried out at the final follow up. The second is that they reflect a degree of psychological destabilisation caused by the occurrence of an unexpected life threatening illness requiring major intracranial surgery. The third explanaton is we believe the most plausible though difficult to prove. The residual symptoms were relatively minor-lassitude, headaches, minor depression-and did not interfere with the patients functional capacity. Such complaints are commonplace in adult life even in persons not receiving medical attention. Their occurrence in our patients may well be no more than would be expexted by chance.

We thank Dr R Blizard, Statistician to the Department of Psychiatry, Royal Free Hospital School of Medicine, for his helpful advice on the statistics.

1 Sotaniemi KA. Five-year neurological and EEG outcome fter open-heart surgery. $J$ Neurol Neurosurg Psychiatry after open-heart

2 Lee WH, Brady MP, Rowe JM, Miller WC. Effects of extracorporeal circulation upon behaviour, personality, and brain function. Ann Surg 1971;173:1013-23.

3 Shaw PJ, Bates D, Cartlidge NEF, Heariside D, Julian DG, Shaw DA. Early neurological complications of coronary artery bypass surgery. BMJ 1985;291:1384-7.

4 Hunt WE, Hess RM. Surgical risk as related to time of intervention in the repair of intracranial aneurysms. $J$ Neurosurg 1968;28:14-9.

5 Storey PB. Psychiatric sequelae of subarachnoid haemorrhage. $B M J 1967 ; 3: 261-6$. 
6 Tessier du Cros J, Lhermitte F. Neuropsychological analysis of ruptured saccular aneurysms of the anterior communicating artery after radical therapy. Surg Neurol 1984;22:353-9.

7 Ljunggren B, Sonesson B, Savelund H, Brandt L. Cognitive impairment and adjustment in patients without neurological deficits after aneurysmal SAH and early operation. J Neurosurg 1985;62:673-9.

8 Storey PB. Brain damage and personality change after subarachnoid haemorrhage. Br J Psychol 1970;117: subarach $129-42$.

9 Bernstein RA, Weir BKA, Petruk KC, Disney LB. Neuropsychological function in patients after subarachnoid psychological function in patients after

10 Matsukado Y, Kitamura K, Shida K, Takamatsu J. Psychiatric disorders after surgery of anterior comunicating chiatric disorders after surgery of anterior comunicating
and internal carotid aneurysms. Brain Nerve 1968;20: and internal carotid aneurysms. Brain Nerve 1968;20:

11 Okawa M, Maeda S, Nukui H, Kawafuchi J. Psychiatric symptoms in ruptured anterior communicating aneurysms: social prognosis. Acta Psychiatr Scand 1980;61:306-12.

2 Logue V, Durward M, Pratt RTC, Piercy M, Nixon WLB. The quality of survival after rupture of an anterior cerebra aneurysm. Br J Psychiatr 1968;114:137-60.

3 Sengupta RP, Chin JSP, Brierley $H$. Quality of surviva following direct surgery for anterior communicating artery aneurysms. J Neurosurg 1975;43:58-64.

14 Fortuny LA, Prieto-Valente L. Long-term prognosis in surgically treated intracranial aneurysms 2: morbidity. $J$ Neurosurg 1981;54:35-43.

15 Takaku A, Tanaka S, Mori T, Suzuki J. Postoperative complications in 1000 cases of intracranial aneurysms. Surg Neurol 1979;12:137-44.

16 Alexander MP, Freedman M. Amnesia after anterior communicating artery aneurysm rupture. Neurology 1984; municating

17 Gade A. Amnesia after operations on aneurysms of the anterior communicating artery. Surg Neurol 1982;18: 46-9. 